\title{
A compact multi-trap optical tweezer system based on CD-ROM technologies
}

\section{T. McMenamin, W. M. Lee}

T. McMenamin, W. M. Lee, "A compact multi-trap optical tweezer system based on CD-ROM technologies," Proc. SPIE 10347, Optical Trapping and Optical Micromanipulation XIV, 1034720 (25 August 2017); doi: $10.1117 / 12.2273674$

Event: SPIE Nanoscience + Engineering, 2017, San Diego, California, United States 


\title{
A compact multi-trap optical tweezer system based on
}

\section{CD-ROM technologies}

\author{
T. McMenamin ${ }^{\mathrm{a}}$, W. M. Lee $\mathrm{a}^{\mathrm{*}}$ \\ ${ }^{a}$ Research School of Engineering, College of Engineering and Computer Science, The Australian National University, North \\ Road, Canberra ACT 2601, Australia
}

\begin{abstract}
We implemented an integrated time sharing multiple optical trapping system through the synchronisation of high speed voice coil scanning lens and laser pulsing. The integration is achieved by using commonly available optical pickup unit (OPU) that exists inside optical drives. Scanning frequencies of up to $2 \mathrm{kHz}$ were showed to achieve arbitrary distribution of optical traps within the one-dimensional scan range of the voice coil motor. The functions of the system were demonstrated by the imaging and trapping of $1 \mu \mathrm{m}$ particles and giant unilamellar vesicles (GUVs). The new device circumvents existing bulky laser scanning systems (4f lens systems) with an integrated laser and lens steering platform that can be integrated on a variety of microscopy platforms (confocal, lightsheet, darkfield).
\end{abstract}

\section{INTRODUCTION}

Optical tweezers have been a major enabling tool for investigating the mechanics of biological systems at a cellular level. They have revealed the forces of kinesin particles, the mechanics of DNA transcription and the strength of cellular membranes ${ }^{1,2}$. The ability of an optical tweezers system to generate multiple trapping sites simultaneously further extends its flexibility and usefulness. Existing methods for multiple trap generation have a significant drawback that often requires extensive optical designs such as $4 \mathrm{f}$ lens system. Previously it has been shown that the integrated laser diode $(\sim 40 \mathrm{~mW})$ and objective lens $(0.6 \mathrm{NA})$ system contained within a DVD system can generate a sufficiently focussed and powerful beam spot to create a $2 \mathrm{D}$ optical trap ${ }^{3}$.In that work, the lens voice coil motor was manually controlled using a variable current source, for single cell sorting in a microfluidic system. However, automation and multiple traps have not been explored using such an integrated system.

In this paper we aim to extend an integrated laser scanning system to achieve multiple distinct optical traps through time sharing as well as demonstrate the small footprint that can be integrated with existing microscope platforms. To achieve multiple distinct optical traps using a time-sharing method, the response of the voice coil motor needs to be consistent for a given electrical input, therefore an experiment was conducted to characterise the position response of the system. As a start, we first measure the repeatability and reliability of the voice coil motor movement. After that we constructed several attachments to include the CD trap and showed its capability to create line traps.

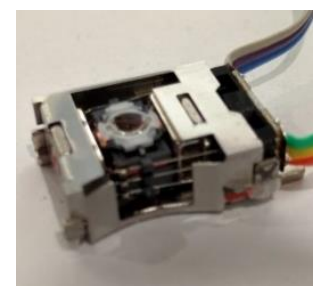

Figure 1 - Voice coil motor (VCM) extracted from a PHR803T optical pickup unit

\section{CALibRATIONS}

For all beam characterisation tests, the experimental setup shown in figure 2.a) was used. The optical pickup was placed vertically such that the beam was focussed at the imaging plane of a two-lens magnification system for detection of small movements. The camera used was a ThorLabs model DCC1645C-HQ CMOS system. The effective magnification of the imaging setup with respect to the CMOS sensor was $0.0187 x$, corresponding to an individual pixel size of $0.067 \mu \mathrm{m}$ at the imaging plane.

*steve.lee@anu.edu.au

Optical Trapping and Optical Micromanipulation XIV, edited by Kishan Dholakia, Gabriel C. Spalding, Proc. of SPIE Vol. 10347, 103472O · C) 2017 SPIE · CCC code: 0277-786X/17/\$18 · doi: 10.1117/12.2273674 
The focal length of the optical pickup was measured by moving the imaging CCD along the focal point of the emitted beam. The measured distance was approximately $2 \mathrm{~mm}$, which corresponds well to the expectation for DVD systems ${ }^{4}$. The beam spot size was determined by centroid tracking. A sample recorded image is shown in figure 2.b) below, and a custom MATLAB image processing algorithm was created for automatic position detection and Gaussian fitting of the emitted beam spot. Analysis of 210 images gathered beam quality information and showed that there was negligible variance in both beam intensity and geometry within the entire field of view of the experimental setup, as shown in figure 2.c). The resolved beam spot size was $0.8 \mathrm{um}$.

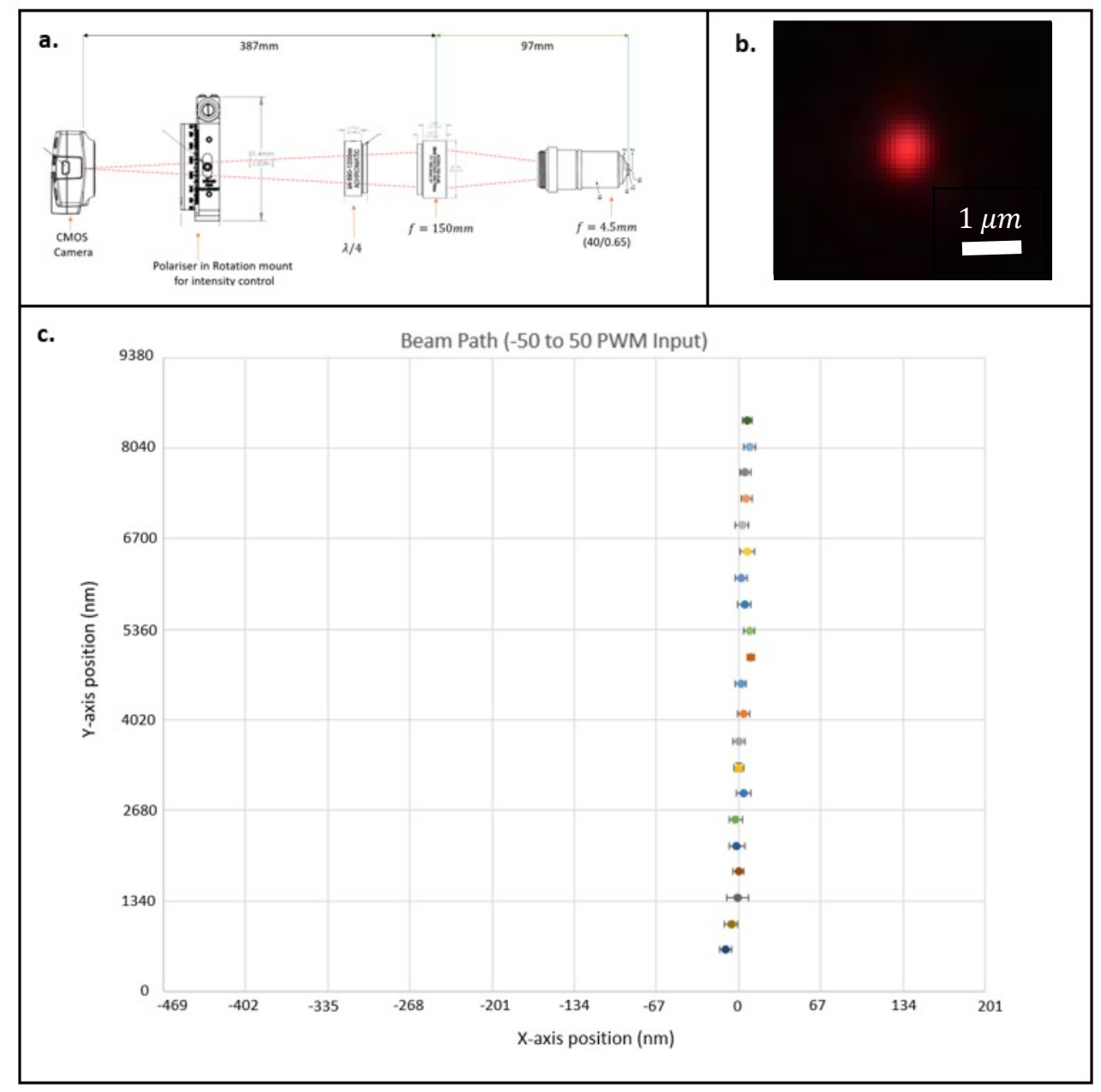

Figure 2 - Beam steering experimental setup and results. (a) optical train used for beam tracking. (b) individual captured data-point of beam location. (c) data resulting from 10 sweeps of the beam across the field of view of the setup, with position extracted by a gaussian fit of beam spot intensity.

This shows the high level of repeatability of the voice coil motor that do not create any significant degradation of beam quality. For time-shared optical traps, a trapped particle experiences a blinking event (absence of the trapping beam) that is related to the scanning frequency of the system. Smaller particles require a higher frequency to remain trapped, and so an increased frequency range allows the system to be used with a wider range of sample sizes 5 . The voice coil can operate at frequencies well above $300 \mathrm{~Hz}$ due to decreased inertia of the scanning component relative to other mirror scanning devices. The frequency response of the voice coil motor system is shown in figure 3 below, gathered using the same optical setup shown in figure 2.a). The scanning range was to operate at frequencies up to $2 \mathrm{kHz}$. We postulate that VCM control voltage (high frequency) can be increased without resistive thermal effects damaging the system for shorter periods of operation ( few seconds). 


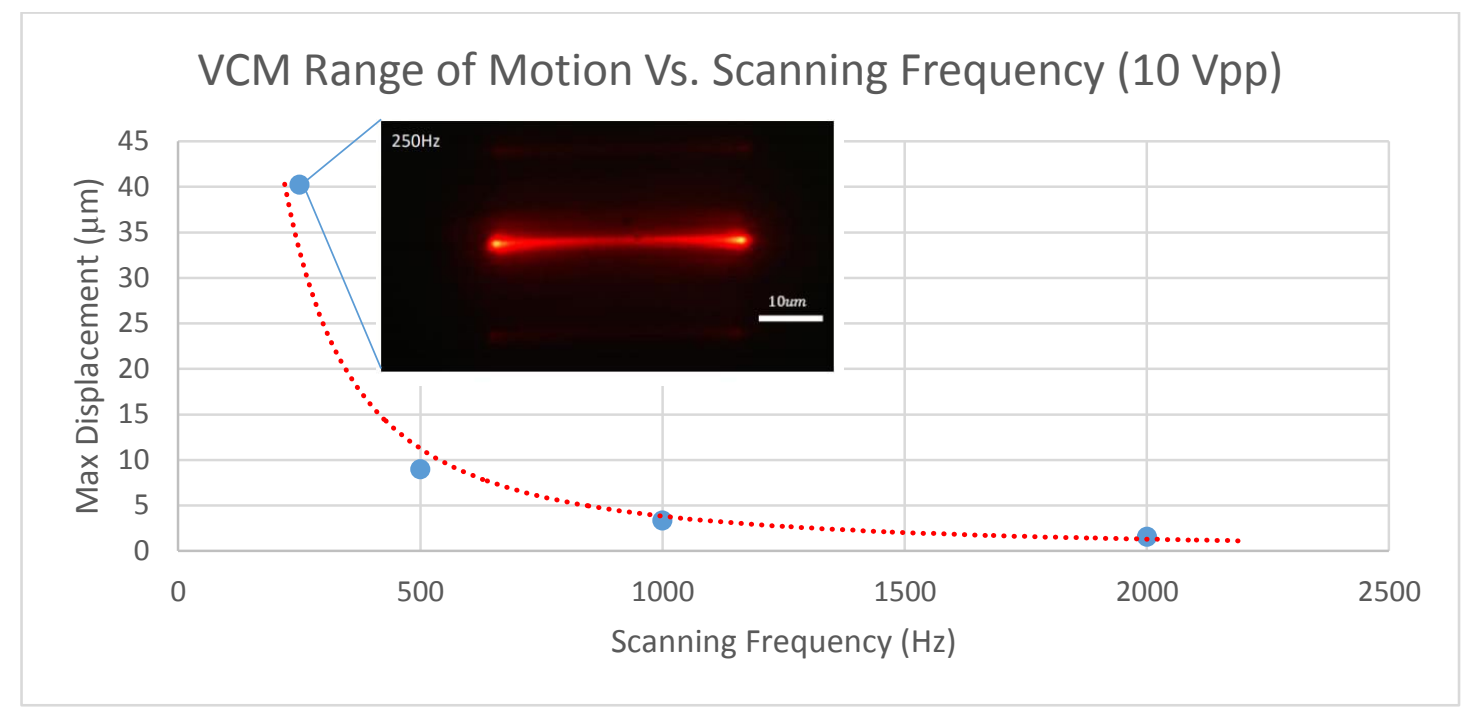

Figure 3 - Maximum displacement amplitude of line scanning beam across different frequencies. (inset) line beam corresponding to a $250 \mathrm{~Hz}$ scanning frequency.

Next, we describe how to transform a rapidly scanning line beam into multiple time-shared optical traps, by pulsing the laser diode during lens scanning. Consider the inset line beam in figure 3, but with the laser diode only active at the ends of the line, this would create two distinct optical traps at those locations. However, the frequency and phase of the laser pulsing signal must be perfectly synchronised with the physical movement of the voice coil beam scanner. This is challenging to realise in practice. Initial attempts to create multiple distinct optical traps via laser pulsing, relied on the manual frequency matching of the laser signal to the voice coil signal via a laboratory function generator. This proved to be unsuccessful due to constant drift between the two signals over time. This drifting manifests in the movement of the optical traps along the line path of the beam scanner, as the laser pulses at different beam positions. This is illustrated in figures 4.a) and 4.b), showing the optical trap drift over the course of 30 seconds. Stable optical traps could not be achieved through this method. Therefore, in order to generate stable time-shared traps, the laser pulsing and voice coil motor signals must be synchronous, such that they have a time-invariant phase relationship. This was achieved through generation of the two signals within the same microcontroller - with the laser diode signal being an even multiple of the VCM driving signal - such that they are tied to the same clock signal generated by an onboard $16 \mathrm{MHz}$ crystal oscillator. As the signals are generated digitally, their relative frequency and phase can be selected as desired such that any $1 \mathrm{D}$ trap pattern can be generated.

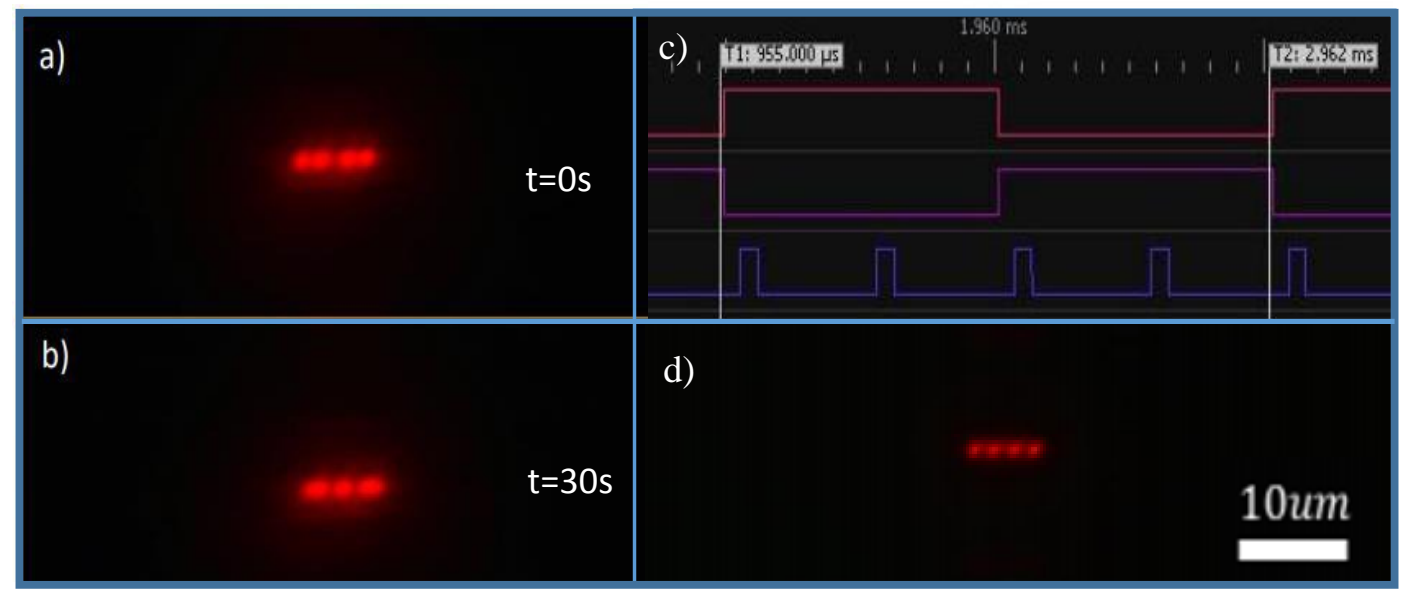

Figure 4 - asynchronous and synchronous laser and VCM control. (a) observed generated beam spot pattern with four pulses at $500 \mathrm{~Hz}$. (b) observed pattern drift over after 30 seconds due to phase changes between the two control signals. (c) synchronous signal generation of VCM signal (top two traces) and Laser signal (bottom trace) from common clock (d) observed beam spot pattern at $500 \mathrm{~Hz}$, stable and invariant over time. 
Upon completion of the synchronous design, and a minor shifting of the relative phase of the laser pulse and voice coil signals, 4 distinct optical traps were able to be achieved at a scanning frequency of $500 \mathrm{~Hz}$ and a laser pulse frequency of $2 \mathrm{kHz}$. The laser and voice coil control signals are shown in figure 4.c), measured experimentally using a high frequency logic analyser, and the resultant traps are shown in figure 4.d). This was the first time that multiple distinct optical traps have been generated using a synchronous lens scanning and laser pulsing method, and demonstrates the potential of our multi-trap design. Due to the change of momentum at the ends of the oscillation, the beam experiences a significant dwell time as it changes direction ${ }^{6}$. This leads to a non-trivial relationship between the laser pulse signal phase and the actual resultant trap position as shown in figure 5.a). This can be visualised effectively by observing the inconsistent intensity of points along the $x$-axis in the corresponding line beam, as can be seen in the inset in figure 2.a).

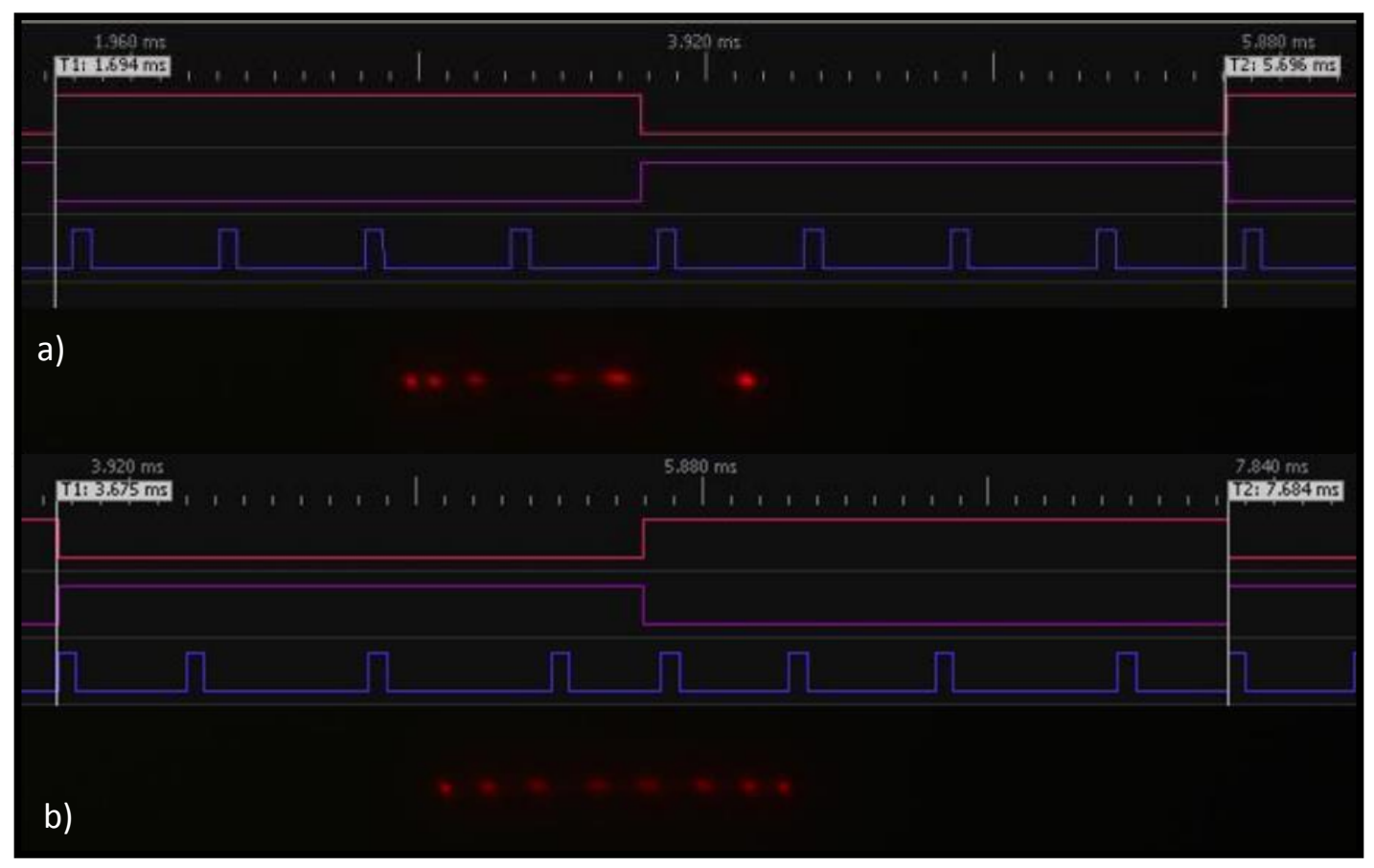

Figure 5 - Velocity compensation for control of trap distribution (a) observed beam pattern when scanning at $250 \mathrm{~Hz}$ with 8 laser pulses at $1 / 8^{\text {th }}$ duty cycle. (b) observed beam pattern when scanning at $250 \mathrm{~Hz}$, with 8 pulses of the same length, but position shifted to compensate for varying velocity of the voice coil motor.

The purpose of the velocity compensation process is to generate the required laser pulse location and duration such that equidistant traps of equal shape and intensity are created. However, it could equally be applied to generation of traps of different separations, shapes and intensities, as desired by any individual application. The trap positions were then corrected by attenuation of the laser diode signal, as shown in figure 5.b) for a $250 \mathrm{~Hz}$ scanning frequency. Examples of different trap generation at various scanning frequencies are shown in figure 6 below, demonstrating the flexibility of the device, as well as giving an indication of trap quality as it relates to different numbers of traps. Note that the power of each individual trap can be calibrated arbitrarily by controlling the amplitude or duty cycle of the laser driving signal for each pulse, such that identical power of each trap can be ensured. 

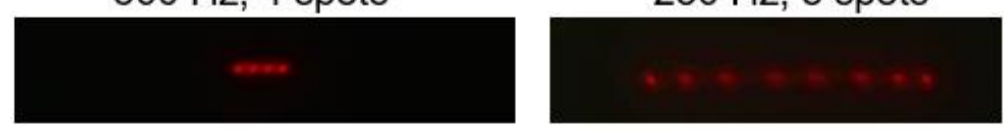

$167 \mathrm{~Hz}, 14$ spots

\section{Multi-trap Beam intensity profile}

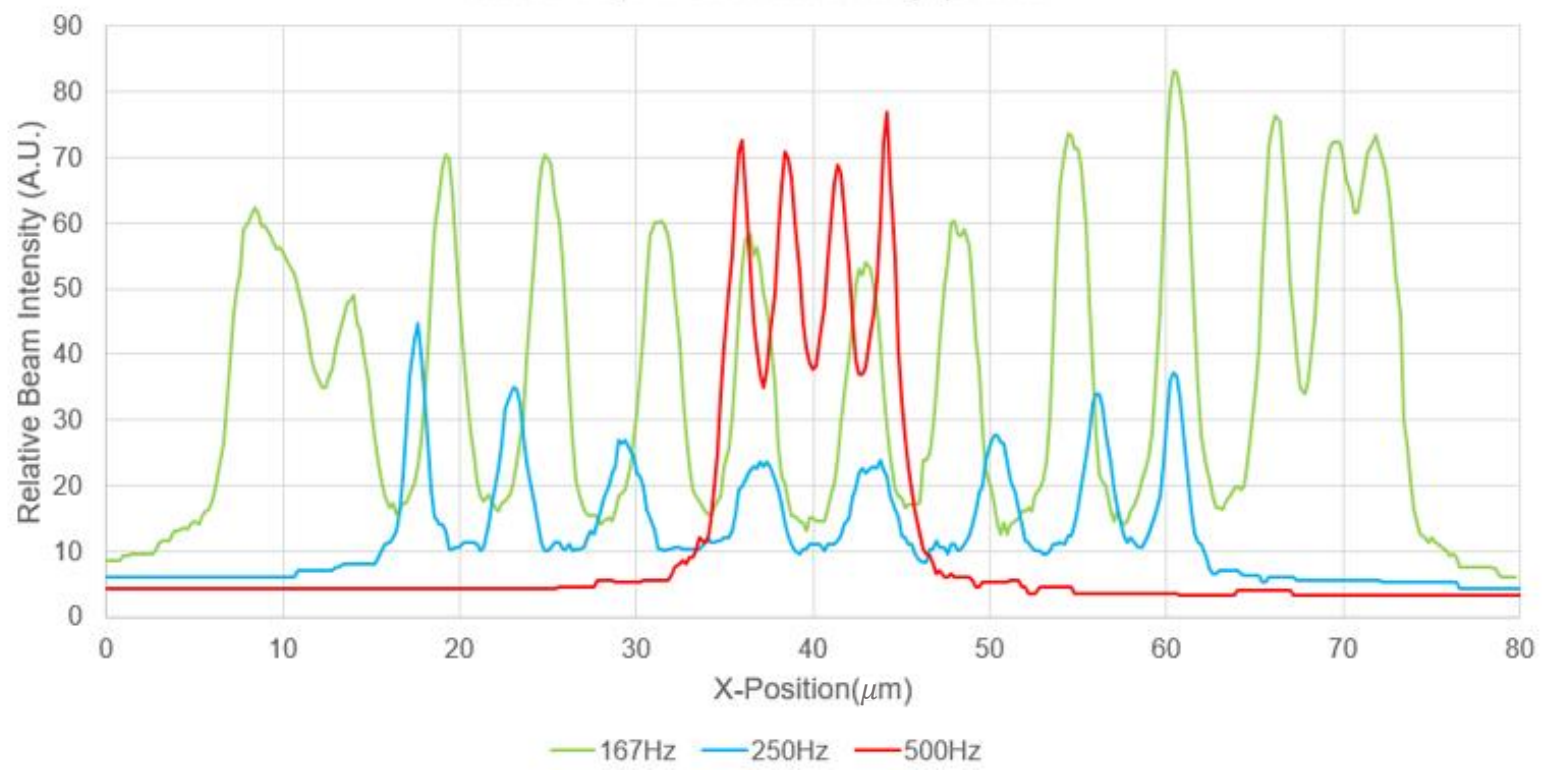

Figure 6 - Observed beam patterns and intensity profiles for traps generated at $500 \mathrm{~Hz}, 250 \mathrm{~Hz}$ and $167 \mathrm{~Hz}$.

\section{APPLICATION}

\section{Darkfield}

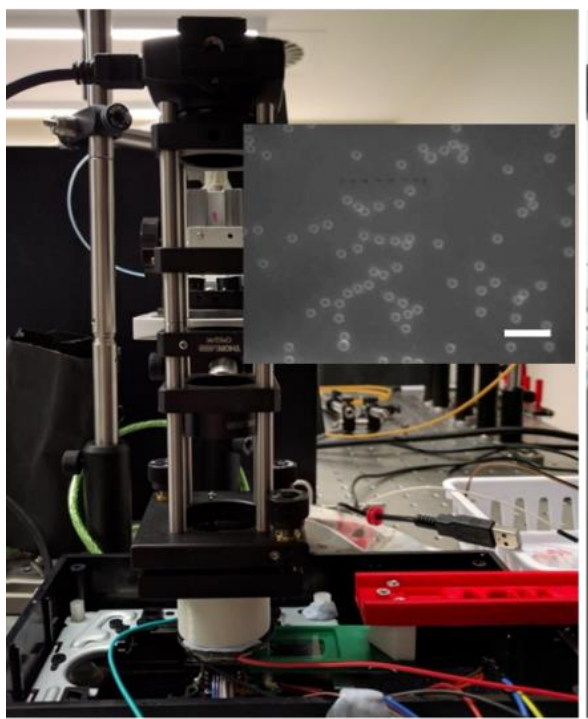

Confocal

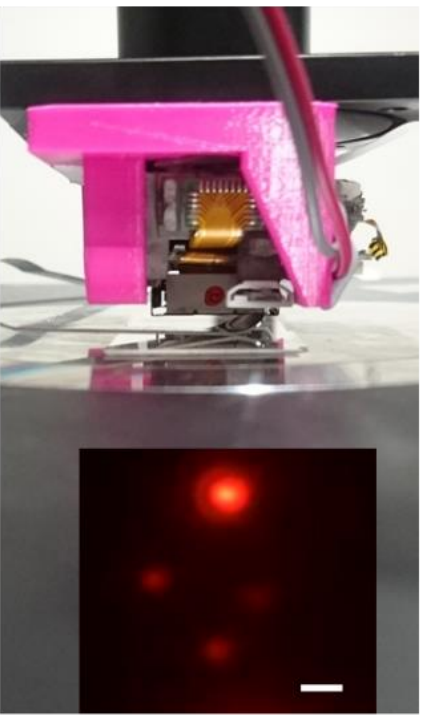

Lightsheet

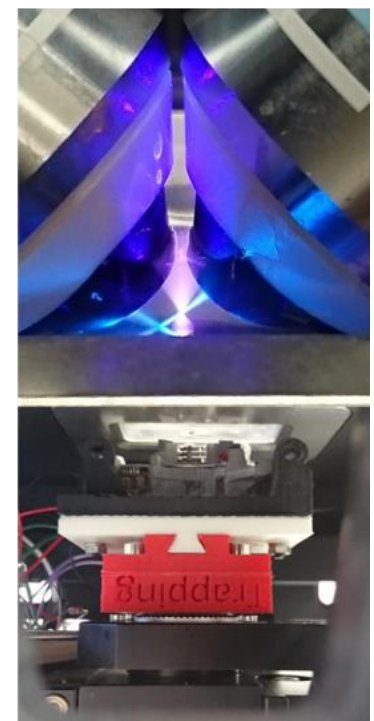

Figure 7 - Integration of the CDROM trapping system into various microscope systems.

Various trapping experiments were conducted using the CDROM-based optical trap. For these experiments, the laser diode was controlled using the ubiquitous LM317-based constant current circuit, and the voice coil motor by a 10Vpp signal of an ATMEGA32u4 AVR microchip, situated on an Arduino prototyping board. The VCM control signal is then amplified for better current handling. Due to the compact nature of our trapping system, it may be easily integrated into various imaging modalities as shown in figure 7. Custom 3D printed mounts have been designed to attach the optical pickup to each microscope, to ensure stability and correct alignment of the trap with the existing imaging objective. The one external optical consideration still required by our system is that the trapping beam needs to be filtered out of the imaging signal. This can be done using any of the established methods, such as a dichroic or fluorescence excitation filter. 


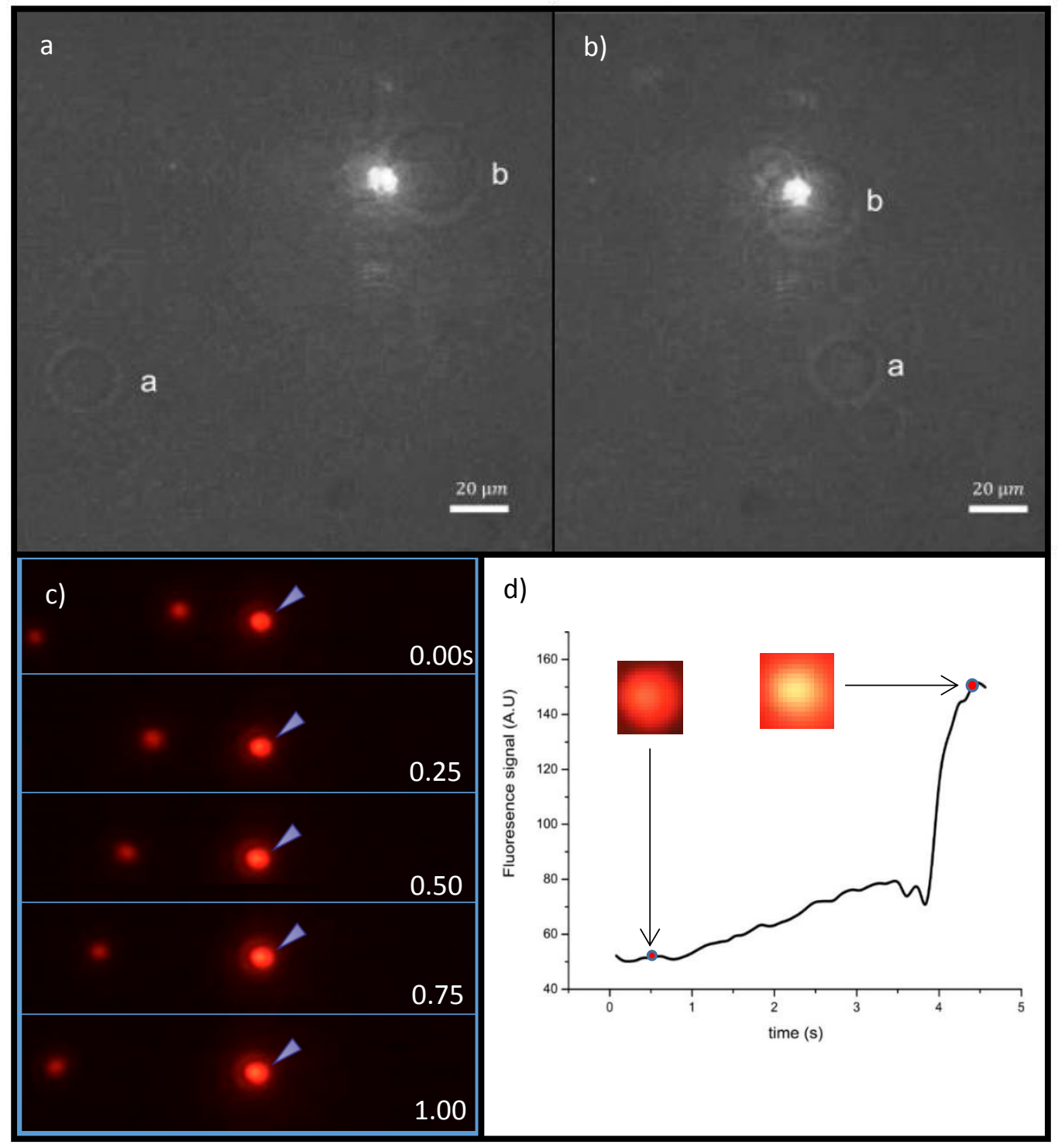

Figure 8 - Single particle trapping experiments. (a) initial position of GUV samples, with particle b trapped by the $650 \mathrm{~nm}$ laser diode (b) final position of GUV samples, after movement of the stage to the right, showing that GUV 'b' was held in place by the optical trap. (c) observation of $405 \mathrm{~nm}$ trapping and fluorescence excitation showing the stationary position of the trapped particles as the sample stage is moved to the left. (d) fluorescence response of the system as a trapping event occurs that increases the number of trapped particles.

Initial testing of the trapping capabilities of our system was carried out using a homebuilt darkfield microscope, which utilises a ring-shaped led array to provide the required illumination angle. Trapping of giant unilamellar vesicles (GUVs) was able to be achieved and imaged using this system, as shown in figure 8.a) and 8.b). GUV ' $\mathrm{b}$ ' was trapped by the CDROM system using the $650 \mathrm{~nm}$ diode, and the sample stage moved without its position being disrupted, this is evident in the change in relative position of the two GUV samples. Experiments were then conducted by integrating our trapping system onto the sample stage of a commercial confocal microscope. By using the $405 \mathrm{~nm}$ diode in our optical pickup unit, which is normally used for Blu-ray/HD-DVD standards, we were able to simultaneously trap and excite fluorescent $1 \mu \mathrm{m}$ microparticles, as shown in figures 8.c) and 8.d).

After experimenting with single trapping using the $650 \mathrm{~nm}$ and $405 \mathrm{~nm}$ wavelengths, line trap experiments were conducted on the same system. Figure 10.a) demonstrates the trapping and alignment of multiple $1 \mu \mathrm{m}$ microparticles in a line beam. The trap stiffness perpendicular to the line scanning axis was then quantified, using the equipartition theorem ${ }^{6}$, as shown in figure 10.b). 

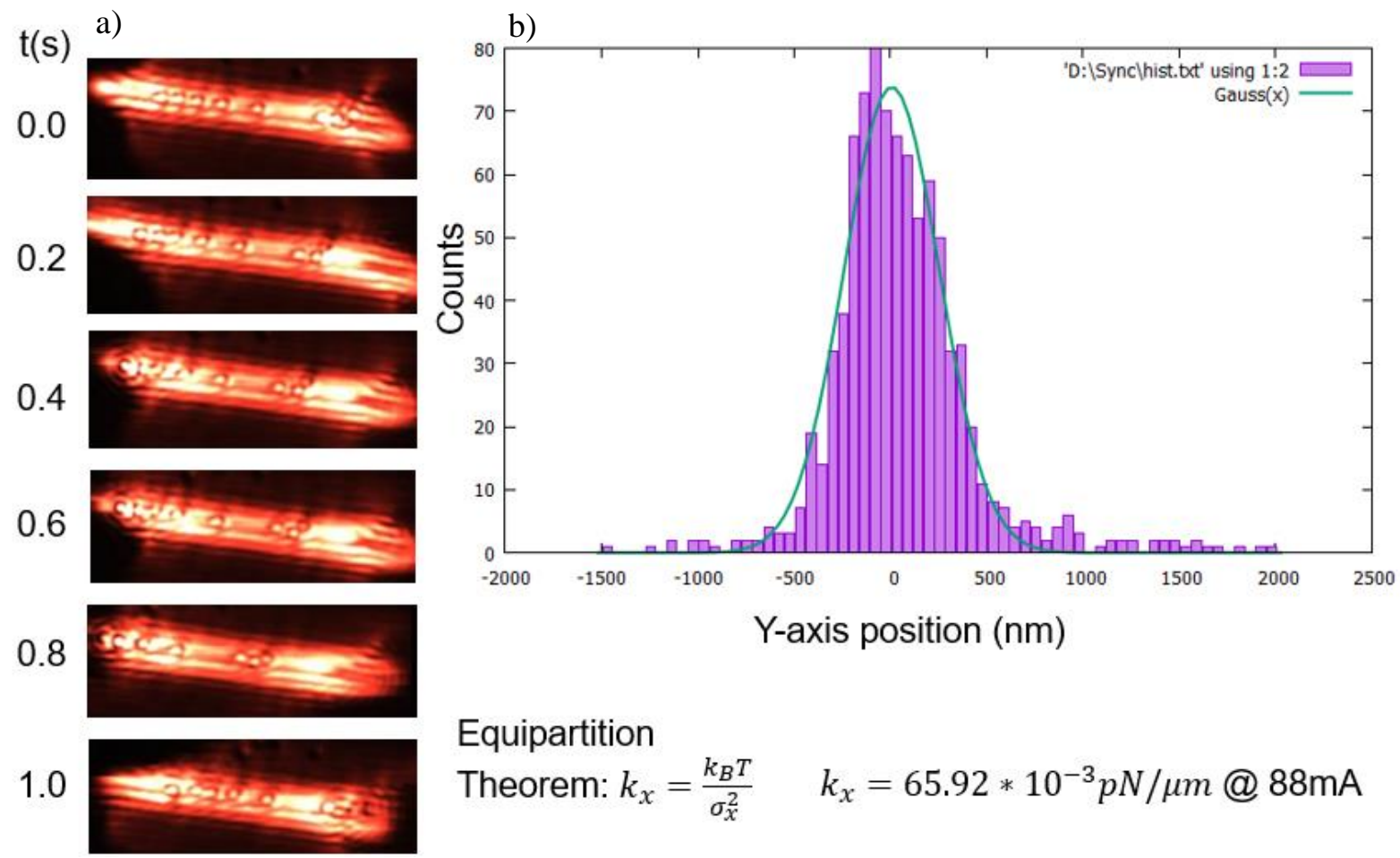

\section{Equipartition \\ Theorem: $k_{x}=\frac{k_{B} T}{\sigma_{x}^{2}} \quad k_{x}=65.92 * 10^{-3} p N / \mu m @ 88 \mathrm{~mA}$}

Figure 9 - Line trapping experiments. (a) observed trapping and alignment of $1 \mu \mathrm{m}$ microparticles in one dimension by line trap. (b) histogram of coordinate values perpendicular to the line trap, with gaussian fit for calculation of trap stiffness.

The calculated trap stiffness is low, as expected. This is a result both of the low NA of the CDROM objective lens (0.6) and the laser intensity being spread out across the line. These experiments show that optical trapping using a CDROM-based system is viable, flexible and accessible. Figure 7 shows the system as integrated into the two aforementioned imaging modalities, as well as on an Applied Scientific Instrumentation (ASI) diSPIM Lightsheet system, where it is screwed into the epifluorescence objective thread using a 3D-printed mount. It has now been shown that multiple distinct optical traps can be generated arbitrarily in one dimension using only the optical components found in the commonly available optical pickup unit used in optical disc drives. By using a time shared approach generated by pulsing the laser diode while the voice coil motor-mounted objective lens is continously scanning, a high degree of stability and control can be obtained over trap position. The varying velocity of the objective lens as it scans is related to its position in a predictable manner, such that the laser pulsing signal can be attentuated such that the desired trap distribution is generated. Experimentation was conducted to demonstrate the viability of single and line beam trapping systems based on this architecture.

\section{CONCLUSION}

We demonstrate for the first time an integrated multiple distinct optical trap system using a synchronous laser pulsing method. We resolved a non-trivial relationship between the laser pulse signal phase and the actual resultant trap position to achieve the potential of multi-trap design. We have developed a control infrastructure for the system based around a single microcontroller board running custom firmware and GUI software, such that the entire system may be implemented for less than 20 USD. 


\section{REFERENCES}

[1] Kuo, S. C. and Sheetz, M.P. "Force of single kinesin molecules measured with optical tweezers," Science 260(5105), 232-234 (1993).

[2] Baumann, C. G., Cross, S. J. "Probing the mechanics of the complete DNA transcription cycle in real-time using optical tweezers," Methods Mol Biol. 778, 175-191 (2011).

[3] Kasukurti, A., Potcoava, M., Desai, S. A., Eggleton, C. and Marr D. W. M., "Single-cell isolation using a DVD optical pickup,” Opt. Express 199(11), 10377-10386 (2011).

[4] Benschop, J. and Van Rosmalen, G., "Confocal compact scanning optical microscope based on compact disc technology,” Appl Opt. 30(10), 1179-1184 (1991).

[5] Čižmár, T., Davila Romero, L. C., Dholakia, K. and Andrews, D. L. "Multiple optical trapping and binding: new routes to self-assembly," J. Phys. B: At. Mol. Opt. Phys. 43(10), (2010).

[6] Borlinghaus, R. T., "Smart control for resonant galvo scanners," Leica Microsystems, 1 August 2014, http://www.leica-microsystems.com/science-lab/smart-control-for-resonant-galvo-scanners/ (7 August 2017). 